Priorities for Care was used or not. To add some narrative to capture the complexity of caring for each individual with a learning disability.

Background This audit evolved as an outcome of a previous learning disabilities audit in January 2015. Following discussion at a multi-disciplinary team meeting it was agreed that a further audit on an individual's PPD would highlight if the hospice was meeting their objectives within the learning disability pathway under future planning considerations.

Method A qualitative retrospective audit was completed from 12 patient's notes on i-care entries, who were known to have a learning disability over a one year period.

Results All deaths achieved their PPD with 100 percent documented evidence. There was a rich narrative and person-centred care, demonstrating continuity.

Conclusion The results revealed aspiring achievements with significant learning outcomes for the hospice multi-disciplinary teams. Actions identified were: An easy read booklet for preferred priorities for care to be offered where appropriate: To highlight the importance of documenting capacity/PPD discussions where appropriate. Current notes to be reviewed with regards to power of attorney/registration. To promote awareness of the resource folder in the clinical nurse specialist office: To share the importance of the narrative and adherence to the standard in the learning disability pathway.

\section{P-127 ANTIMICROBIAL STEWARDSHIP - IMPROVING PRESCRIBING ON THE INPATIENT UNIT}

Wendy Pepper, Rebecca Bhatia. Dorothy House Hospice Care, Bradford-On-Avon, UK

\subsection{6/bmjspcare-2016-001245.150}

Introduction The Inpatient Unit at Dorothy House Hospice Care committed to comply with national guidelines for antibiotic prescribing. The document followed was the Department of Health's 'Antimicrobial Stewardship: Start Smart Then Focus'. Although the guidance had been produced for hospitals the guiding principles were also applicable in the hospice setting. We also wanted to ensure compliance with Criterion 3 of the Health and Social Care Act 2015 where it states "Ensure appropriate antimicrobial use to optimise patient outcomes and to reduce the risk of adverse events and antimicrobial resistance".

Aims The aims were to ensure Dorothy House was compliant with local and national guidelines on antibiotic prescribing

To identify how antibiotics are prescribed and supplied by Dorothy House and to improve antimicrobial stewardship.

Methods A patient database was set up to capture all patients prescribed antibiotics.

Standards were set to ensure:

- Compliance with local guidelines

- Documented indication for antibiotic use was on the medicine chart

- Documented indication for use was in the patient notes

- The prescription was clear and accurate

- A review date or duration of antibiotic use was prescribed

- Compliance with The Department of Health Advisory Committee on antimicrobial stewardship

- A quarterly audit using the above standards was performed capturing all patients prescribed antibiotics in that time period
Conclusion There was a dramatic improvement in compliance from the quarter one audit to the quarter four audit in all standards but most specifically around documenting an indication for use on prescription charts and setting a review or duration date. There was also a decrease in the use of broad spectrum antibiotics. This audit now forms part of the Dorothy House Hospice Care annual audit plan.

\section{P-128 "EN SUITE DRUGS": QUALITY IMPROVEMENTS IN MEDICINES MANAGEMENT}

Tara Schrikker. St Wilfrid's Hospice, Eastbourne, UK

\subsection{6/bmjspcare-2016-001245.151}

Background The hospice introduced the practice for controlled and non-controlled drugs to be stored and administered from the Patient Own Drug (POD) cupboards located within patients' bedrooms. Further recommendations were addressed after initial implementation to strengthen patient safety.

Aims and objectives

What was the aim of the work?

Increase Productivity and Clinical Effectiveness

For timely medication rounds as staff remain in the patients' rooms at point of administration.

Enhanced Patient Experience

Staff remaining within the patients' rooms provides a visible, reassuring presence.

Quality Improvement and Safety

For stock levels to be tailored to patient need ensuring cost effectiveness

Storing patient's own controlled drugs in PODS reduces the risk of administration incidents.

Method

- Proposal presented to Clinical Governance Committee to introduce PODs in patients' rooms, storing controlled and non-controlled medication.

- Medicines Management Group guided implementation

- Clinical Commissioning Group (CCG) informed of the proposed changes in practice, including the financial implications.

- POD cupboards redesigned and controlled drug cabinets installed to meet legislation and compliance.

- Training sessions held to support staff during change to practice.

- Second phase of implementation included administration of oral controlled drugs from PODs.

Results

- Clinical staff have reported improvement with timely medication rounds.

- Audits found a reduction in the time between the actual prescribed time and patients receiving their oral modified release opioids.

- Patient focused to allow continuation of their preferred administration times.

- Promotion of enabling patients to retain control and independence of their medications.

- Improvement in the management of drug stock levels.

- Decrease in number of related medication incidents in comparison to the previous year.

Conclusion This quality improvement involved all levels of the hospice team. The successful implementation required 
engagement with stakeholders, including the CCG. The focus of improved patient experience, safety, and clinical effectiveness was maintained. The innovation allowed a greater focus on enabling patients' to maintain their independence in managing their medicines.

\section{P-129 THE ESSENCE OF TIME - CAN AUTOMATED DISPENSING RELEASE HOSPICE NURSING TIME FOR PATIENT CARE?}

Tracy Livingstone, Nan Jones. Nightingale House Hospice, Wrexham, UK

\subsection{6/bmjspcare-2016-001245.152}

While there is research supporting the benefit of automated dispensing for the prevention of medication errors in emergency departments Fanning et al. (2015) this has not previously been looked at in a hospice setting neither has the impact of automated dispensing and top-up on releasing nurses time to spend with patients in this area.

With the likelihood of increasing demand for hospice services and increasing complexity of patient needs there is a drive to provide more care from the same or even less resource, technology ranging from telemedicine and remote monitoring to automated systems may provide an opportunity to increase our ability to meet these challenges and free up nurses to focus on the provision of patient contact and care that hospices are historically associated with.

This presentation follows the journey within a 12-bedded inpatient hospice of installation of automated dispensing medication system in a hospice in-patient unit, from the point of pre installation time and motion studies of medication dispensing through installation and beyond investigating the impact on:-

- System acceptability to staff

- Nurse time taken to dispense

- Nurse time taken to complete stock checks and weekly top-up

- Nurse satisfaction with system following installation

- pre and post installation medication error rates.

In addition the presentation reflects the benefit of considering change proactively rather than as a response to critical incidents with medication and the significant impact this can have on staff confidence and morale within a small team.

\section{P-130 "JUST IN CASE" BAGS - WHAT IS THE VALUE OF ANTICIPATORY PRESCRIBING ON DISCHARGE FROM AN ACUTE TRUST?}

\footnotetext{
1,2 Helen Lock, ${ }^{2}$ Simon Haslam, ${ }^{2}$ Alice King, ${ }^{2}$ Caroline Pinckney, ${ }^{2}$ Rameshwor Sunar, ${ }^{2}$ Jennifer Barry, ${ }^{1,2}$ Becky Baines. ${ }^{1}$ Hospiscare, Exeter, UK; ${ }^{2}$ Royal Devon and Exeter NHS Foundation Trust
}

\subsection{6/bmjspcare-2016-001245.153}

The concept of "Just in Case" bags to support anticipatory prescribing in end of life care is not new, but there is little research evidence to support their use - anecdote and small audit level evidence at best. The recent 2015 NICE guidance on care of the dying patient highlighted the lack of evidence and specifically suggested more research into this topic.

In our rural community (population 380,000), "Just in Case" bags have been used in practice for many years. Over the last two years, it has been usual practice to dispense small supplies of "Just in Case" medication on discharge from our large, 75bedded acute trust.

The usefulness of Just in Case bags is being reviewed in a number of ways:

1. Retrospective review of hospital and specialist palliative care records of a cohort of patients discharged over a six-month period with "Just in Case" medication

2. Attempts are being made to determine appropriateness, usage and effectiveness of symptom control

3. Estimation of costs

4. Preferred and actual place of death

5. Detailed review of patients prescribed "renal friendly" drugs checking for appropriateness and effectiveness.

An analysis of problems associated with Just in Case bags will be undertaken and attempts to improve the process will be undertaken using quality improvement methodology.

The work is being developed by a team of junior doctors in the Trust, with the support of the specialist palliative care lead consultant and registrar. We also aim to show that by working closely with the lead prescribers of these medications (junior doctors) we can highlight the appropriate use and potential benefits of "Just in Case" bags in a more systematic way. At the same time we hope that this project will enhance more general awareness of end of life care beyond the acute hospital in this key group of staff.

\section{P-131 ENHANCING MEDICINES SAFETY - SMALL CHANGES LEAD TO BIG IMPROVEMENTS!}

${ }^{1}$ Kate Marley, ${ }^{1}$ Carole Slocombe, ${ }^{1}$ Clare Doyle, ${ }^{2}$ Graham Holland. 'Woodlands Hospice, Liverpool, UK; ${ }^{2}$ Liverpool Heart and Chest Hospital

\subsection{6/bmjspcare-2016-001245.154}

Introduction Woodlands Hospice has a transparent approach to incident reporting and staff are encouraged to report near misses. In 2014/15, 76 medicines administration errors were reported, many relating to incomplete documentation. 'Enhancing medicines safety and reducing documentation errors' was chosen as an organisational priority for 2015/16.

Actions Improvements were led by the hospice's Medicines Management Group. A revised medicines management policy was implemented and the annual medicines training programme revised.

Inpatient nurses were consulted for their ideas about improvements in medicines safety and 'fact-finding' visits were made to local hospices. All practical ideas were considered and the following were implemented:

- A 'Woody' sign (based on the hospice rabbit mascot) was designed as an aide-memoire to be placed on bedroom doors to indicate that a medicines-related action needs to be completed e.g. return to administer heparin

- An additional medicine trolley was purchased to reduce the number of patients on each medicine round

- A Controlled Drug checklist was devised to ensure daily completion of documentation.

Results Medicines administration incidents for the year 2015/16 reduced from 76 to 25 .

Additional benefits included: 